\title{
Les Determinants des Changements Sociaux Lies a la Gouvernance de l'eau Autour du Perimetre Rizocole de Malanville au Nord est du Benin
}

\author{
Dine S. Assouma, \\ Nouroudine Ollabode, \\ Jacob Afouda Yabi,
}

Département d'Economie et Sociologie Rurales, Faculté d'Agronomie, Université de Parakou

Doi:10.19044/esj.2019.v15n21p304 URL:http://dx.doi.org/10.19044/esj.2019.v15n21p304

\section{Résumé}

Description du sujet : Pour analyser les rapports sociaux induits par la gestion de l'eau dans la production agricole, la plupart des études mettent l'accent sur l'action conjointe ou concertée, la coopération et les conflits avec des effets émergents. Objectifs : La présente recherche a pour objectif d'analyser les changements sociaux liés à la gouvernance de l'eau autour du périmètre irrigué de Malanville. Méthode : À travers une série d'enquêtes socioéconomiques auprès de 210 chefs d'exploitations rizicoles, les informations relatives aux types de rapports en fonction des caractéristiques sociodémographiques ont été collectées. L'analyse des données collectées sur la base d'un questionnaire a été faite à l'aide d'un logiciel SPSS21 et une régression logistique a été réalisée afin d'identifier les déterminants du changement social des enquêtés. Les théories de l'action collective ont été également utilisées pour analyser les données. Résultats : Les résultats ont montré que la gestion de l'eau est devenue un enjeu politique, économique et juridique considérable qui s'exprime à travers des stratégies d'affrontement, de coopération et de concertation. Conclusion : Ainsi, le mode de gestion de l'eau, le respect des règles de l'UGPPM, le mode de distribution de l'eau, l'accès aux crédits, la superficie totale emblavée, les aiguadiers comme acteurs de conflit, la période du conflit (saison sèche) et l'occupation de poste sont des déterminants dans les changements sociaux.

Mots clés: Changements sociaux, eau, gouvernance, périmètre irrigué, Malanville 


\title{
The Determinants of Social Change Related to the Governance of Water Around the Rizocole Perimeter of Malanville in Northeast Benin
}

\author{
Dine S. Assouma, \\ Nouroudine Ollabode, \\ Jacob Afouda Yabi,
}

Département d'Economie et Sociologie Rurales, Faculté d'Agronomie, Université de Parakou

\begin{abstract}
To analyze the social relations induced by water management in agricultural production, most studies focus on joint or concerted action, cooperation and conflicts with emerging effects. The present research aims to analyze the social changes related to governance around the irrigated area of Malanville. Through a series of socio-economic surveys of 200 farm managers, information on the types of reports based on socio-demographic characteristics was collected. The analysis of the data collected on the basis of a questionnaire was done using an SPSS21 software and a logistic regression was carried out in order to identify the determinants of the social change of the respondents. Theories of collective action were also used to analyze the data. The results showed that water management has become a considerable political, economic and legal issue, expressed through strategies of confrontation, cooperation and consultation. Thus, the mode of water management, the respect of the rules of the UGPPM, the mode of distribution of the water, the access to the credits, the total area planted, the aiguadiers like actors of conflict, the period of the conflict (dry season) and post occupation are determinants in social change.
\end{abstract}

Keywords: Social change, Water, Governance, Irrigated area, Malanville

\section{Introduction}

En Afrique de l'Ouest, la pénurie en eau, est entrain de connaître une intensification très importante qui mettra l'agriculture pluviale en déclin dans le futur. La demande croissante en eau est confrontée à une diminution significative de l'offre de la ressource provoquée par les variabilités climatiques (Houdret, 2009a). Ces variabilités climatiques se manifestent par 
une modification du régime des précipitations et par une diminution de la hauteur pluviométrique annuelle (Alomasso, 2018). Les fluctuations climatiques menacent la disponibilité des ressources en eau par une forte concurrence, le développement des activités socioéconomiques des populations participent à leur dégradation et constituent de ce fait des facteurs de risques qui fragilisent la santé des populations et la sécurité alimentaire en eau (Houdret, 2009b; Alomasso, 2018).

$\mathrm{Vu}$ l'ampleur des variabilités climatiques, l'irrigation des superficies de production agricole parait indispensable pour la croissance économique des pays subissant les effets du changement climatique. Selon Bzioui (2004), l'agriculture irriguée assure la stabilité du produit intérieur brut car l'agriculture détermine une part importante dans le PIB. Force est de constater que des conflits parfois violents surviennent de plus en plus fréquemment entre usagers de l'eau autour des périmètres irrigués dus surtout à un manque de gouvernance (UNESCO/WWAP $2003: 2 ; 2006: 3$ ).

Dans les pays en développement (PED), la gouvernance des ressources en eau constitue sans doute un enjeu politique, économique et social majeur (BiedCharreton, 2006). Le service de l'eau est un enjeu politique au sens le plus noble du terme car gérer l'eau, c'est d'abord choisir entre différentes contraintes et opportunités, environnementales, sociales et économiques (Frérot, 2011).

L'eau est devenue un bien économique du fait qu'elle est rare. Plusieurs institutions internationales se mobilisent autour de cette denrée de première nécessité dont la pénurie s'explique en partie par l'accroissement rapide de la population de la planète en ces dernières décennies. Cela rejoint le fait que l'abondance de l'eau dans une zone lui confère un excellent potentiel agricole, maillon du développement, cas des zones tropicales humides.

L'enjeu social s'explique par le fait que la gouvernance de l'eau conduit à des conflits et à l'exclusion de certaines couches de la population.

La gouvernance de l'eau constitue donc un enjeu de plus en plus central pour le management des organisations, qu'elles soient publiques ou privées (Casteigts, 2000). Une gouvernance appropriée favorise la répartition équitable de l'eau entre les usagers tout en assurant la protection du patrimoine commun de la nation (loi sur l'eau de 1992). Elle doit permettre à toutes les parties d'exprimer leurs points de vue et d'être entendues et dans le même temps d'être un cadre favorable à la protection de cette ressource (Lanfranchi, 2008). La présente étude a pour objectif d'analyser les déterminants des changements sociaux liés à la gouvernance de l'eau autour du périmètre irrigué de Malanville. 


\section{Collecte des donnes \\ 1.1. Zone d'étude}

La zone d'étude est le périmètre rizicole irrigué de Malanville se trouvant dans la vallée du Niger qui va de Guéné jusqu'au lit mineur du fleuve et constitue un espace géographique doté d'atouts pour l'édification d'une régionalisation économique cohérente et viable. Situé dans le Nord-Est du Bénin, sous un climat soudano-sahélien avec l'alternance d'une saison pluvieuse (mi-octobre) et d'une saison sèche (novembre-avril), le périmètre est d'une importance socio-économique pour les coopérateurs. D'une superficie de 516 ha répartis en dix quartiers Hydrauliques de taille variable, il est le plus grand des périmètres irrigués du Bénin.

\subsection{Base des données collectées}

Les unités d'observation sont les producteurs du périmètre irrigué rizicole de la zone d'étude. Deux cent dix (210) chefs d'exploitations rizicoles échantillonnés au hasard ont été enquêtés. La constitution de l'échantillon a été raisonnée (choix guidé des producteurs) et aléatoire (sélection au hasard des riziculteurs). Le principal instrument de collecte utilisé pour recueillir les données relatives aux coûts et revenus de la production était un questionnaire individuel administré aux producteurs échantillonnés à travers des entretiens individuels. De même, des "focus group" ont été organisés pour croiser les données telles que : intrants agricoles, quantité d'eau utilisée, équipements, et main d'œuvre utilisée. Enfin, des observations et la triangulation des informations ont été faites pour s'assurer de la qualité des informations recueillies.

\subsection{Modèle}

\section{La spécification du modèle}

Le changement social est estimé par un modèle de probabilité basé sur la décision du producteur pour laquelle cette décision est fonction des caractéristiques du changement social du producteur. L'identification des facteurs qui influencent la décision d'opérer un changement social passe le plus couramment par les modèles Probit, Logit et Tobit (Makokha et al, 2003). Les modèles Probit et Logit sont utilisés lorsque la variable dépendante est dichotomique et Tobit est employé à la fois pour la modélisation de la décision d'adoption et à la détermination de l'intensité d'utilisation de la technologie. Pour des raisons de commodité, le modèle Logit est le plus souvent utilisé dans la modélisation des décisions d'adoption de nouvelle technologie (CIMMYT, 1993). Dans notre étude, le modèle Logit a été retenu et adapté au changement social qu'opère le producteur lorsque les éléments du fonctionnement de l'organisation sociale connaissent une variation. Ce modèle a été choisi car il 
est plus adapté pour déterminer les facteurs qui influent le changement social lié à la gouvernance de l'eau.

La densité de Bernoulli du changement social s'écrit : $\mathrm{f}(\mathrm{yi})=$ $\mathrm{p}^{\mathrm{y}_{\mathrm{i}}}\left(1-\mathrm{y}_{\mathrm{i}}\right)^{1-\mathrm{y}_{\mathrm{i}}} ; \mathrm{y}_{\mathrm{i}}$ représente la variable dépendante et $p$ la probabilité du changement social. Ainsi, $y_{i} \sim B(1, P)$.

Si $\mathrm{y}_{\mathrm{i}}=1$, le changement social est la coopération;

Si $\mathrm{y}_{\mathrm{i}}=0$, le changement social est le conflit

$y_{i}=F\left(\beta^{\prime} X_{i}\right)+\epsilon_{i}$. L'espérance mathématique $\mathrm{E}\left(\mathrm{y}_{\mathrm{i}}\right)=\sum_{\mathrm{i}=0}^{1} \mathrm{p}_{\mathrm{i}} \mathrm{y}_{\mathrm{i}}$

Alors $\mathrm{E}\left(\mathrm{y}_{\mathrm{i}}\right)=F\left(\beta^{\prime} X_{i}\right)$ car l'espérance des erreurs est nulle.

$\epsilon_{i}=y_{i}-F\left(\beta^{\prime} X_{i}\right)$. Ainsi, $\mathrm{E}\left(\epsilon_{i}\right)=\sum_{\mathrm{i}=0}^{1} \mathrm{p}_{\mathrm{i}}\left[y_{i}-F\left(\beta^{\prime} X_{i}\right)\right]=0 \leftrightarrow=F\left(\beta^{\prime} X_{i}\right)$

Le changement social résulte de toute transformation observable dans le temps, qui affecte, d'une manière en n'étant pas que provisoire ou éphémère, le fonctionnement de l'organisation sociale d'une collectivité donnée (Macquaire, 2018). Avant de dire qu'il y a un changement social, le producteur compare le déclin des anciennes appartenances sociales et l'apparition de nouvelles identités plus valorisées que les anciennes relatives au divers fonctionnement de l'association auquel il appartient. Le changement social peut-être positif ou négatif. Il est positif lorsqu'il y a coopération, concertation, équité et négatif lorsqu'il y a disparité, inégalité, rancunes, guerre froide, conflit dans la gouvernance de l'eau. Ces éléments du fonctionnement de l'organisation sociale peuvent se modifier et traduire un changement social pendant que ces informations restent privées et détenues par le producteur. Lorsque nous considérons une variable qui puisse prendre en compte les informations privées détenues par le producteur, une telle variable est qualifiée de variable latente ou discrète et est continue.

Il a été conclu que la plupart des études des déterminants sont considérées comme l'adoption d'une nouvelle technologie avec une mesure discrète. Ainsi, le changement social est la coopération lorsqu'il y a la concertation, l'équité, l'attente entre les riziculteurs dans la gouvernance de l'eau autour des périmètres irrigués. Le changement social est le conflit lorsqu'il y a la disparité, l'inégalité, les rancunes, et la guerre froide dans la gouvernance de l'eau. En effet, le changement social a été mesurée de façon discrète.

Soit $\mathrm{y}^{*}$ la variable latente ou discrète représentant le changement social (coopération et conflit).

$\mathrm{Y}^{*}=\beta^{\prime} X_{i}+\epsilon_{i}$ avec $\beta^{\prime}=$ le vecteur des paramètres à estimer, $X_{i}$ représente les variables explicatives, $\epsilon_{i}$ les termes d'erreurs.

On a les cas suivants :

* Si $\mathrm{Y}^{*}>0$ alors le changement social est la coopération, donc $y_{i}=1$

* Si $\mathrm{Y}^{*} \leq 0$ alors le changement social est le conflit, $y_{i}=0$ 
Considérons le cas où le changement social est la coopération $\left(y_{i}=1\right)$. Alors la probabilité $\mathrm{p}$ est donnée par :

$p\left(y_{i}=1\right)$ signifie $p\left(\mathrm{Y}^{*}>0\right)=p\left(\beta^{\prime} X_{i}+\epsilon_{i}>0\right)$ alors, $p\left(\epsilon_{i}>-\beta^{\prime} X_{i}\right)=$ $F\left(\beta^{\prime} X_{i}\right)$.

On fait l'hypothèse que les erreurs $\epsilon_{i}$ sont indépendantes et identiquement distribuée et suivent la loi logistique.

La modélisation logistique de $\mathrm{y}$ en fonction de $\mathrm{p}$ variables explicatives $\left(X_{1}, X_{2}, \ldots, X_{p}\right)$ et de $\mathrm{p}+1$ paramètres à estimer $\left(\beta_{0}, \beta_{1}, \beta_{2}, \ldots, \beta_{p}\right)$ est :

$p\left(y=1 \backslash X_{1}=x_{1}, X_{2}=x_{2}, \ldots, X_{p}=x_{p}\right)=\frac{e^{\beta_{0}+\sum_{i=1}^{p} \beta_{i} x_{i}}}{1+e^{\beta_{0}+\sum_{i=1}^{p} \beta_{i} x_{i}}}$

La fonction de lien logistique devient: $\operatorname{logit}\left(y=1 \backslash X_{i}=x_{i}\right)=\beta_{0}+$ $\sum_{i=1}^{p} \beta_{i} x_{i}, i=\overline{1, n}$

En introduisant le terme d'erreur, on obtient le modèle économétrique suivant :

$$
\begin{aligned}
\operatorname{logit}\left(y=1 \backslash X_{i}\right. & \left.=x_{i}, i=\overline{1, n}\right)=\beta_{0}+\beta_{1} x_{1}+\beta_{2} x_{2}+\cdots+\beta_{p} x_{p}+\epsilon_{i} \\
\mathrm{Y}_{\mathrm{ij}}=\beta_{0}+\beta_{1} & \text { ANEPRij }+\beta_{2} \text { ACRij }+\beta_{3} \text { CONFONASSij }+\beta_{4} \text { CONFONASSij } \\
& +\beta_{5} \text { RESUGPPMij }+\beta_{6} \text { PERCONFLITij }+\beta_{7} \text { ACTCONFLIij } \\
& +\beta_{8} \text { SUPTij }+\beta_{9} \text { MOFTij }+\beta_{10} \text { ACHEAUij }+\beta_{11} \text { PAYDROIij } \\
& +\beta_{12} \text { POSTOCUPij } \\
& +\beta_{13} \text { APPREij }+\beta_{14} \text { MOGEAUij }+\beta_{15} \text { MODEAUij }+\epsilon_{i}
\end{aligned}
$$

\begin{tabular}{|c|c|c|c|}
\hline Variables & Types $^{\mathbf{a}}$ & Modalités & Signes attendus \\
\hline Expérience dans la production du riz (ANEPR) & $\mathrm{C}$ & - & - \\
\hline Accès aux crédits agricole (ACR) & $\mathrm{D}$ & $0=$ Non, $1=$ Oui & - \\
\hline $\begin{array}{l}\text { Connaissance du fonctionnement de l'association } \\
\text { (CONFONASS) }\end{array}$ & $\mathrm{D}$ & $0=$ Non, $1=$ Oui & \pm \\
\hline Respect des règles de l'UGPPM (RESUGPPM) & $\mathrm{D}$ & $0=$ Non, $1=$ Oui & + \\
\hline Période des conflits (PERCONFLI) & $\mathrm{D}$ & $0=$ Non, $1=$ Oui & \pm \\
\hline Acteurs des conflits (ACTCONFLI) & $\mathrm{D}$ & $0=$ Non, $1=$ Oui & \pm \\
\hline Superficie totale emblavée (SUPT) & $\mathrm{C}$ & - & + \\
\hline Main d'œuvre familiale totale (MOFT) & $\mathrm{C}$ & - & + \\
\hline Accès à l'eau (ACHEAU) & $\mathrm{D}$ & $0=$ Non, $1=$ Oui & + \\
\hline Payement des droits d'adhésion (PAYDROI) & $\mathrm{D}$ & $0=$ Non, $1=$ Oui & \pm \\
\hline Occupation d'un poste (POSTOCUP) & $\mathrm{D}$ & $0=$ Non, $1=$ Oui & + \\
\hline Application des règles du fonctionnement de & $\mathrm{D}$ & $0=$ Non, $1=$ Oui & - \\
\hline l'association (APPRE) & $\mathrm{D}$ & $0=$ Gestion & \pm \\
\hline Mode de gestion de l'eau (MOGEAU) & & $\begin{array}{l}\text { déléguée, } \\
\text { 1=Gestion } \\
\text { collective }\end{array}$ & \\
\hline Mode de distribution de l'eau (MODEAU) & $\mathrm{D}$ & $\begin{array}{c}0=\text { Station de } \\
\text { pompage } \\
1=\text { Motopompe }\end{array}$ & - \\
\hline
\end{tabular}

Tableau 1:Variables introduites dans le modèle Logit 
${ }^{\mathrm{a}}: \mathrm{D}=$ variables Discontinues $\mathrm{C}=$ variables Continues

\section{Résultats}

\section{Statistiques descriptives des caractéristiques des changements sociaux}

Les statistiques descriptives des caractéristiques des changements sociaux des enquêtés sont résumées dans le tableau 2. Les résultats d'analyse révèlent que l'activité agricole demeure la principale activité masculine (92\% des riziculteurs sont des hommes et $8 \%$ sont des femmes) dans la zone d'étude. $49 \%$ des riziculteurs ont accès aux crédits pour la production du riz et $57 \%$ de ces riziculteurs ont un contact avec un service de vulgarisation. La majorité des riziculteurs $(60 \%)$ ont accès à l'eau sur les sites de production. Le payement des droits d'adhésion est fait par (57,5\%) des différents membres de l'association. Il faut noter que $40 \%$ des enquêtés avouent avoir occupé un poste dans le bureau de l'association. L'application des règles du fonctionnement de l'association et le respect des règles de l'UGPPM sont faits pour $66 \%$ et $65,5 \%$ des enquêtés respectifs. $69 \%$ des enquêtés ont confirmé que cette forme de gestion de l'eau peut-être durable. Il faut souligner que deux modes de gouvernance sont utilisées sur les périmètres irrigués à savoir : mode de distribution et mode de gestion de l'eau. La gestion déléguée (69,5\%) et la gestion collective (30,5\%) sont les deux types de mode de gestion de l'eau utilisées par les riziculteurs. Quant au mode de distribution, la station de pompage $(90 \%)$ et la motopompe (10\%) sont les deux types de mode de distribution de l'eau utilisée sur les sites de production autour du périmètre irrigué.

Le changement social s'opère sous deux formes au sein des associations dans la gouvernance de l'eau sur le périmètre irrigué dans la commune de Malanville. Il s'agit du Conflit et de la coopération. Lorsqu'il y a conflit ou coopération entre les différents membres on parle de changement social lié à la gouvernance de l'eau. Sur ce, $35,5 \%$ des enquêtés ont déclaré qu'il y a conflit contre $64,5 \%$ qui ont affirmé qu'il y a coopération dans la gouvernance de l'eau sur les périmètres irrigués. Pour ceux qui ont avoué qu'il existe de conflit, les acteurs responsables de ces conflits sont les riziculteurs $(60,5 \%)$ et les Aiguadiers (39,5\%). Les riziculteurs sont les principaux acteurs causant les conflits. Les causes de ces conflits sont la corruption (32,5\%), l'utilisation frauduleuse de l'eau (21\%) et le détournement de l'eau pour servir un quartier non programmé $(46,5 \%)$. Ces conflits naissent plus pendant la saison sèche (Tableau). En dehors des causes évoquées par les enquêtés, la connaissance ou non du fonctionnement de l'association est aussi l'une des causes des conflits ou de la coopération au sein de l'association. 
Tableau 2 : Statistiques descriptives des caractéristiques des changements sociaux

\begin{tabular}{|c|c|c|c|c|c|}
\hline Variables & Modalités & $\begin{array}{c}\text { Fréquenc } \\
\text { es }\end{array}$ & Variables & Modalités & Fréquences \\
\hline \multirow[t]{2}{*}{ Accès aux credits } & Non & 51 & \multirow[t]{2}{*}{ Accès à l'eau } & Non & 60,0 \\
\hline & Oui & 49 & & Oui & 30,0 \\
\hline \multirow{2}{*}{$\begin{array}{l}\text { Contact avec un } \\
\text { service de } \\
\text { vulgarisation }\end{array}$} & Non & 43,0 & \multirow{2}{*}{$\begin{array}{l}\text { Durabilité de la } \\
\text { forme de gestion } \\
\text { de l'eau }\end{array}$} & Non & 31,0 \\
\hline & Oui & 57,0 & & Oui & 69,0 \\
\hline \multirow[t]{2}{*}{ Accès à l'eau } & Non & 60,0 & \multirow{2}{*}{$\begin{array}{l}\text { Payement des } \\
\text { droits d'adhésion }\end{array}$} & Non & 42,5 \\
\hline & Oui & 30,0 & & Oui & 57,5 \\
\hline \multirow{2}{*}{$\begin{array}{l}\text { Occupation d'un } \\
\text { poste dans } \\
\text { l'association }\end{array}$} & Non & 60,0 & \multirow{2}{*}{$\begin{array}{l}\text { Application des } \\
\text { règles du } \\
\text { fonctionnement } \\
\text { de l'association }\end{array}$} & Non & 34,0 \\
\hline & Oui & 40,0 & & Oui & 66,0 \\
\hline \multirow[t]{2}{*}{$\begin{array}{l}\text { Mode de gestion de } \\
\text { l'eau }\end{array}$} & $\begin{array}{l}\text { Gestion } \\
\text { déléguée }\end{array}$ & 69,5 & \multirow[t]{2}{*}{$\begin{array}{l}\text { Respect des règles } \\
\text { de l'UGPPM }\end{array}$} & Non & 34,5 \\
\hline & $\begin{array}{l}\text { Gestion } \\
\text { collective }\end{array}$ & 30,5 & & Oui & 65,5 \\
\hline \multirow[t]{2}{*}{$\begin{array}{l}\text { Mode de } \\
\text { distribution de l'eau }\end{array}$} & $\begin{array}{l}\text { Station de } \\
\text { pompage }\end{array}$ & 90,0 & \multirow[t]{2}{*}{ acteurs des conflits } & $\begin{array}{l}\text { Producteur } \\
\text { s }\end{array}$ & 60,5 \\
\hline & Motopompe & 10,0 & & Aiguadiers & 39,5 \\
\hline \multirow[t]{2}{*}{ Période des conflits } & $\begin{array}{l}\text { Saison } \\
\text { pluvieuse }\end{array}$ & 41,0 & \multirow[t]{2}{*}{$\begin{array}{l}\text { Changement } \\
\text { social }\end{array}$} & Conflit & 35,5 \\
\hline & Saison sèche & 59,0 & & $\begin{array}{c}\text { Coopératio } \\
\text { n }\end{array}$ & 64,5 \\
\hline \multirow[t]{3}{*}{$\begin{array}{l}\text { Connaissance du } \\
\text { fonctionnement de } \\
\text { l'association }\end{array}$} & Non & 18,0 & \multirow[t]{3}{*}{$\begin{array}{l}\text { Cause des } \\
\text { conflits }\end{array}$} & $\begin{array}{c}\text { Utilisation } \\
\text { frauduleus } \\
\text { e de l'eau }\end{array}$ & 21 \\
\hline & \multirow[t]{2}{*}{ Oui } & 82,0 & & $\begin{array}{l}\text { Détournem } \\
\text { ent de l'eau }\end{array}$ & 46,5 \\
\hline & & & & Corruption & 32,5 \\
\hline
\end{tabular}

Source : Résultats d'analyse d'enquêtes de terrain, 2018.

\subsection{Déterminants du changement social lié à la gouvernance de l'eau}

Des résultats obtenus, plusieurs variables ont une incidence tant positive que négative sur la décision du changement social liés à la gouvernance de l'eau autour du périmètre irrigué de Malanville au NordBénin. Les résultats montrent que le modèle estimé est globalement statistiquement significatif. En effet, le log vraisemblance $(36,339)$ est satisfaisant, ainsi que le test d'indépendance de Khi-deux du modèle $\left(\chi^{2}=31,569\right)$ qui est significatif à $1 \%$. Cela stimule qu'il y a une forte corrélation entre les variables (Pseudo $\mathrm{R} 2=0,475$ ). Les variations des variables explicatives incluses dans le modèle expliquent à $47,5 \%$ les variations des changements sociaux liés à la gouvernance de l'eau autour du périmètre irrigué de Malanville. De plus, au niveau des paramètres estimés, on trouve globalement neuf (9) variables ayant une influence significative sur la 
probabilité du changement social opéré dans la gouvernance de l'eau. Ces variables sont entre autres :

1. L'accès aux crédits agricoles influence négativement les changements sociaux liés à la gouvernance de l'eau au seuil de 5\%. Les riziculteurs ayant accès aux crédits agricoles ont été plus responsables des conflits naissant dans la gestion de l'eau. Cet état de choses pourrait s'expliquer par le fait que ces riziculteurs n'ont pas acquis des moyens nécessaires auprès des IMFs pour s'épargner des pénuries de l'eau au sein de l'exploitation agricole. Ils ont eu à s'opposer aux différents systèmes de gouvernance lorsqu'ils seront en pénuries d'eau car ils ne pourront pas rembourser leur crédit à la fin des campagnes si le rendement est mauvais, vu que le riz est une culture exigeante en eau.

2. Le respect des règles de l'UGPPM détermine positivement le changement social lié à la gouvernance de l'eau au seuil de $5 \%$. Cela induit que plus les règles de l'UGPPM sont respectées et appliquées, plus les riziculteurs coopèrent au sein de l'association et moins les conflits sont nés dans la gouvernance de l'eau. Les membres de l'association se concertent et s'entendent pour des décisions communes dans la gestion de l'eau du périmètre irrigué.

3. La période des conflits : Saison sèche est déterminante dans les changements sociaux liés à la gouvernance de l'eau au seuil de 5\%. La connaissance de la période des conflits permet aux membres de l'association de coopérer ensemble pour établir des règles de collecte, de distribution de l'eau à l'avance afin de minimiser les conflits liés à la gouvernance de l'eau pendant la saison sèche qui est la période de naissance des conflits puisque la culture aura besoin plus de l'eau pour sa croissance.

4. Les acteurs de conflits : Aiguadiers, déterminent négativement le changement social lié à la gouvernance de l'eau à 5\%. Ils sont responsables des divers conflits nés autour de la gestion de l'eau. Du fait qu'ils sont chargés de distribuer l'eau aux membres de l'association, ils le font par affiliation, connaissance, liens parentaux, et parfois ils sont corrompus par les riziculteurs.

5. La superficie totale emblavée par les riziculteurs induit négativement le changement social donc il y a de conflit autour de la gouvernance de l'eau. Plus la superficie est grande, plus la demande en eau est élevée et plus les riziculteurs mettront des actions fortes à l'égard des aiguadiers (négociation, détournement, corruption, etc.) pour avoir l'eau nécessaire au moment opportun pour les plants du riz.

6. L'Accès à l'eau a une influence positive à $10 \%$ sur le changement social dans la gouvernance de l'eau. Du coup, plus le riziculteur a accès à l'eau, plus il y a la coopération entre les membres de l'association et moins les 
conflits naissent. Par conséquent, la répartition de l'eau au sein de l'association a été faite de façon équitable.

7. L'Occupation d'un poste dans le bureau au sein de l'association affecte positivement le changement social dans la gouvernance de l'eau à $5 \%$. Ceci peut s'expliquer par le sérieux que met cette personne dans le fonctionnement du périmètre, la rigueur dans l'application des règles du mode de gouvernance et plus il amène les membres à coopérer pour la bonne gouvernance de l'eau.

8. Le mode de gestion de l'eau impacte positivement le changement social à 5\% cela peut s'expliquer par le fait que la gestion de l'eau est faite par l'application des règles établies et aussi par la conservation de l'eau pour son utilisation au moment de décru. Plus la gestion est collective, plus il y a de la coopération dans la gouvernance de l'eau. Plus les riziculteurs coopèrent pour la gestion de l'eau moins il y a des conflits car les riziculteurs gèrent mieux l'eau en coopérative parce qu'il y a des règles préalablement établis au sein de leur coopérative auxquels les membres ont l'obligation de respecter et d'appliquer.

9. Le mode de distribution de l'eau a un impact négatif à $5 \%$ sur le changement social. Cela peut s'expliquer par le fait que la distribution de l'eau est faite par affinité et aussi par le manque d'expérience dans la programmation de distribution de l'eau au moment de décru. Par conséquent, le mode de distribution affecte le conflit comme changement social dans la gouvernance de l'eau.

En outre, les autres variables caractérisant le changement social introduites dans le modèle ont des effets marginaux les plus forts mais non significatives. En plus, certaines variables affectent positivement et d'autres négativement la propension du changement social dans la gouvernance de l'eau. Une analyse singulière des variables ayant des effets significatifs sur la probabilité de choix nous a permis de mettre en évidence ces effets.

Tableau 3 : Récapitulatif des variables introduites dans la régression logit sur le changement social

\begin{tabular}{lccc}
\hline Variables & Coefficients & Erreurs Standards & $\mathbf{P}>|\mathbf{z}|$ \\
\hline Expérience dans la production du riz (ANEPR) & $-0,069$ & 0,054 & 0,200 \\
Accès aux crédits agricole (ACR) & $-8,053^{* * *}$ & 3,837 & 0,036 \\
Connaissance du fonctionnement de l'association & $-2,253$ & 1,741 & 0,196 \\
(CONFONASS) & & \\
Respect des règles de l'UGPPM (RESUGPPM) & $3,051^{* *}$ & 1,423 & 0,032 \\
Période des conflits (PERCONFLI) & $3,680^{* *}$ & 1,868 & 0,049 \\
Acteurs des conflits (POSTOCUP) & $-5,544^{* *}$ & 2,909 & 0,057 \\
Superficie totale emblavée (SUPT) & $-1,568^{* *}$ & 0,704 & 0,026
\end{tabular}


Main d'œuvre familiale totale (MOFT)

$\begin{array}{ccc}-0,001 & 0,001 & 0,483 \\ 5,034 * & 2,785 & 0,071 \\ -1,732 & 1,225 & 0,157 \\ 5,449 * * & 2,615 & 0,037 \\ -1,367 & 1,673 & 0,414\end{array}$

Application des règles du fonctionnement de l'association (APPRE)

Mode de gestion de l'eau (MOGEAU)

$22,968 * * \quad 11,304 \quad 0,042$

Mode de distribution de l'eau (MODEAU)

$-19,808 * *$

9,356

0,034

Constante

3,964

2,766

0,152

\begin{tabular}{ccc}
\hline & Log vraisemblance & 36,339 \\
Chi-deux & 31,569 \\
Résumé du modèle & Probabilité $>$ chi2 & 0,005 \\
& Pseudo R2 & 0,475 \\
Dll & 14 \\
N* : valeur significative à $5 \%(0,01<\mathrm{P} \leq 0,05) ; *$ : valeur significative à $10 \%(0,05<\mathrm{P} \leq 0,10)$. & 200 \\
\multicolumn{2}{c}{ Source $:$ Résultat d'analyse d'enquête, 2018} &
\end{tabular}

\section{Discussions}

Les résultats de cette étude révèlent que les variables telles que accès aux crédits agricoles, les aiguadiers, la superficie totale emblavée et le mode de distribution de l'eau induisent un changement social négatif qui est le conflit dans la gouvernance de l'eau autour du périmètre irrigué. Ces résultats confirment ceux de Houdret (2009b) selon lesquelles une disparité importante est observée dans les zones rurales aménagées en grande hydraulique au Maroc et le reste du pays, en ce qui concerne la gouvernance de l'eau. Le mode de gestion de l'eau, l'occupation d'un poste, et le respect des règles de l'UGPPM sont des mécanismes mis en place pour assurer la bonne gouvernance de l'eau. Ces résultats corroborent ceux de Houdret (2009a) qui a obtenu que certains mécanismes de répartition ne posent souvent pas de problèmes majeurs dans la gestion de l'eau puisque, même avec une attribution prioritaire pour certains riziculteurs, il reste toujours suffisamment d'eau pour satisfaire la demande des autres et leur assurer un revenu, quoique souvent modeste. Par conséquent les riziculteurs au sein des grands périmètres irrigués se trouvent alors dans une situation relativement favorisée. Ces résultats confirment ceux de Frerot (2011) dans le sens que la distribution et la gestion de l'eau sont orientés vers les buts que les communautés locales ont établis. L'occupation d'un poste affecte positivement le changement social et 
cela se traduit par le fait que, pour Casteigts (2000) l'individu peut contribuer à donner l'orientation qu'il souhaite à la gouvernance de l'eau tout en concourant en proportion des pouvoirs dont il dispose pour jouer un rôle dans la décision collective qui porte sur le coût et la quantité des biens liés à cette gouvernance. Les désaccords ou les conflits autour de l'eau portent alors plutôt sur la question de la mauvaise application des règles et l'ignorance des textes dans la gouvernance de l'eau autour du périmètre irrigué.

\section{Conclusion}

Les changements sociaux que peut induire la gouvernance de l'eau est le conflit et la coopération. Les mesures d'adaptation à la bonne gouvernance de l'eau pouvant induire un changement social positif nécessitent des transformations considérables qui doivent remettre probablement en cause beaucoup de règles établies pour le bon fonctionnement de l'association et la bonne gouvernance de l'eau autour du périmètre irrigué.

\section{References:}

1. Alomasso A. A., 2018. Conflits d'usage dans le bassin béninois du fleuve Niger en Afrique de l'ouest dans le contexte de la gestion intégrée des ressources en eau. Thèse de doctorat, Géographie et Gestion de l'Environnement, Université d'Abomey-Calavi, 220 pages.

2. Annabelle H.. Les conflits autour de l'eau au Maroc: origines sociopolitiques et écologiques et perspectives pour une transformation des conflits.(Konflikte um Wasser in Marokko: ökologische und soziopolitische Konfliktursachen sowie Möglichkeiten der Konflikttransformation). Science politique. Université Paris VIII Vincennes-Saint Denis; Universität Duisburg-Essen, 2008. Français. <tel-00409020>

3. Assouma D., Ollabode N., Issaka K., et Yabi A. J., 2019. Performance économique des producteurs sous divers systèmes de gestion et de distribution de l'eau dans le périmètre rizicole de Malanville. Communication à la $1^{\text {ère }}$ conférence internationale sur enjeux et perspectives économiques en Afrique Francophone à l'université Cheikh Anta Diop de Dakar, du 4 au 6 février 2019.

4. Casteigts M., 2000. La gouvernance environnementale, entre management des organisations et management des territoires. Le duff r. Et rigal j.-j. Maire et environnement, menaces ou opportunités?, Dalloz, pp.163 - 175, 2000. <halshs-01590791>.

5. Frérot A., 2011. Gestion de l'eau, vers de nouveaux modèles. fondapol, fondation pour l'innovation politique 28 pages.

6. Houdret A. Quelle gouvernance des crises sociales liées à la gestion de l'eau? In : Eaux, pauvreté et crises sociales [en ligne]. Marseille : 
IRD Éditions, 2009 (généré le 06 janvier 2019). Disponible sur Internet : <http://books.openedition.org/irdeditions/4827>. ISBN : 9782709917667. DOI : 10.4000/books.irdeditions.4827.

7. Lanfranchi G., 2008. Gouvernance de l'eau à l'échelle du bassin versant français : état des lieux et dispositifs d'évaluation, AgroParisTech - ENGREF à Montpellier, 28 pages.

8. Macquaire P., 2018. Un essai de transformation sociale, le quartier Picassiette à Chartres, Paris, L'Harmattan,

9. Maddison, D. 2006. The perception and adaptation to climate change in Africa. CEEPA. Discussion Paper No. 10. Centre for Environmental Economics and Policy in Africa, University of Pretoria, South Africa.

10. Marc B.-C., Raoudha M., Olivier P., Mélanie R.D., 2006. « La gouvernance des ressources en eau dans les pays en développement : enjeux nationaux et globaux », Mondes en développement 2006/3 (no 135), p. 39-62. DOI 10.3917/med.135.0039 